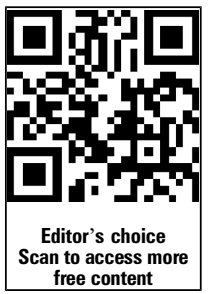

Division of Neurological Surgery, Barrow Neurological Institute, St. Joseph's Hospital and Medical Center, Phoenix, Arizona, USA

\section{Correspondence to}

Dr Nicholas Theodore, Division of Neurological Surgery, Barrow Neurological Institute, St. Joseph's Hospital and Medical Center, 350 W. Thomas Road; Phoenix, AZ 85013, USA Neuropub@dignityhealth.org

Accepted 21 April 2015 Published Online First 18 May 2015
CrossMark

To cite: Ropper AE, Neal MT, Theodore N. Pract Neurol 2015:15:266-272.

\title{
Acute management of traumatic cervical spinal cord injury
}

\author{
Alexander E Ropper, Matthew T Neal, Nicholas Theodore
}

\begin{abstract}
Patients with acute cervical spinal cord injury present complex clinical challenges. These injuries may result in motor and sensory deficits and also in cardiovascular and respiratory perturbations. Increased attention to critical care support has led to improved survival and recovery in many patients. The methods and technology used to diagnose and classify these injuries as well as medical and surgical treatments have evolved significantly in recent decades. We review important aspects of the diagnosis and acute care of patients with traumatic cervical spinal cord injuries, emphasising the recent evidence.
\end{abstract}

\section{INTRODUCTION}

Spinal cord injury (SCI) can be a devastating disease that presents multiple challenges in both acute and chronic phases. Managing patients with SCIs requires a multidisciplinary team that usually includes a neurosurgeon or orthopaedic surgeon, neurologist and physiotherapist. Although our ability to treat injuries surgically has advanced in recent years from a standpoint of spinal column stabilisation, the overall motor and sensory recovery in patients with a severe SCI has not changed. Extensive research is focussing on improving outcomes using stem cells and other adjuvant therapies, such as direct electrical stimulation. ${ }^{1}$ As this research comes to affect the field, we review the basic diagnostic and treatment paradigms reflected in the field's vast literature.

\section{EPIDEMIOLOGY}

The US National SCI Statistics Center estimated the number of people living with SCI in the USA as $273000 .^{2}$ Nearly half of these injuries occurs in young people (aged 16-30 years). Published reports of SCI incidence in the USA vary from 25 to 59 new cases per million population per year with an average of 40 per million, translating to approximately 12400 new SCI cases in $2010 .{ }^{3}$ In the UK and Ireland, it is estimated that 50000 people are living with SCI with an annual health care cost of more than $£ 1$ billion. ${ }^{4}$ Falls are a common cause of injury in the elderly, whereas motor vehicle crashes, violence and sports are the common causes of SCI in children and the younger adult populations. $^{3}$

While it is possible for an SCI to occur in any region of the spine, we choose here to focus on cervical spine injury. Injury to the cervical spine can be the most severe SCI-aside from possible quadriplegia, breathing can be impaired from neurological injury above C5. The cervical spine is especially vulnerable to injury given the relative axial alignment of the facet joints, which require less force to dislocate compared with the thoracic or lumbar spine. In addition, the neck has relatively little external support -compared with the thoracic spine that has the rib cage for stabilisation-predisposing the cervical spine to injury. Although this review focuses mainly on the cervical spine, we also discuss general principles that apply to traumatic thoracic fractures. Significant trauma to the lumbar spine is less common and results in root injuries, which are neurologically distinct from cervical and thoracic trauma.

\section{PREHOSPITAL IMMOBILISATION}

The treatment of any trauma patient begins before the person reaches the hospital. Between $3 \%$ and $25 \%$ of spinal cord injuries occur after the initial trauma, either during transportation or early in the course of management. ${ }^{5}$ The probability of a non-contiguous spinal injury in the setting of a known injury is approximately $20 \%,{ }^{5}$ necessitating the need for complete spinal immobilisation 
for any suspected SCI. Although there is no class I or II evidence supporting the use of a rigid cervical collar in suspected spinal trauma, there are clear anatomical, anecdotal and biomechanical advantages to limiting neck movement in these circumstances. The older practice of head immobilisation with sandbags and tape on a backboard should be used in selected cases of occipitocervical dislocation (once diagnosed) but offers no advantage in most other cervical spine injuries.

There does not appear to be a major difference in the biomechanical constraints of different rigid cervical collars in cadaveric studies. ${ }^{6}$ We believe that a rigid collar should be applied to the neck with manual in-line stabilisation while minimising cervical spine displacement. Spinal immobilisation is a priority of the prehospital trauma algorithms and leads to an improved outcome. ${ }^{7}$ However, class II evidence suggests that patients with penetrating trauma who had prehospital spinal immobilisation have a worse outcome. ${ }^{8}$ Because the process of immobilisation delayed life-saving resuscitation, patients in the study who were immobilised had nearly twice the morbidity and mortality as those penetrating trauma patients who did not have full immobilisation. While cervical collars and backboards provide an important tool to reduce further neurological injury, they have their own inherent risks and complications. Among these are high risks of aspiration, ${ }^{9}$ pressure sores ${ }^{10}$ and increased intracranial pressure. ${ }^{11}$ Practitioners must recognise these risks and understand the need to remove immobilising devices as soon as safety allows.

\section{NEUROLOGICAL ASSESSMENT}

Clinicians must be familiar with the broadly used terminology and scales for grading SCI. International standards for neurological and functional classification of SCI, developed by the American Spinal Injury Association (ASIA), are the recommended preferred neurological examination tool. We summarise these below. ${ }^{12}$

- ASIA A=complete. No sensory or motor function is preserved below the level of injury or in the sacral segments S4-S5.

- ASIA B=incomplete. Sensory but not motor function is preserved below the neurological level and includes the sacral segments S4-S5.

- ASIA C =incomplete. Motor function is preserved below the neurological level, and more than half of key muscles below the neurological level have a muscle grade $<3$.

- ASIA $\mathrm{D}=$ incomplete. Motor function is preserved below the neurological level, and at least half of key muscles below the neurological level have a muscle grade $\geq 3$.

- ASIA E $=$ normal. Sensory and motor function is normal.

The ASIA Scale is simple and has a high interobserver correlation; it is therefore commonly used in care of the acutely injured patient.
Several other scales have been proposed as tools to follow patients with SCI and their progress in rehabilitation. We believe that the Spinal Cord Independence Measure $(\mathrm{SCIM})^{13}$ is a superior descriptor for clinicians involved in rehabilitation medicine. The SCIM is a disability scale developed for patients with spinal cord lesions that makes the functional assessments of patients with paraplegia or tetraplegia more sensitive to changes of recovery. The SCIM focuses on areas of function: self-care (subscore 0-20), respiration and sphincter management (0-40) and mobility (0-40). The SCIM has undergone several iterative revisions: the latest version, the SCIM III, is supported by class I evidence for its reliability, validity and sensitivity. ${ }^{14}$

\section{RADIOLOGICAL ASSESSMENT}

There has been substantial investigation into the types of imaging to be obtained for trauma patients to evaluate possible traumatic spinal pathology. The Joint Section on Disorders of the Spine and Peripheral Nerves of the American Association of Neurological Surgeons and the Congress of Neurological Surgeons have done an excellent service by summarising the vast data in the literature regarding radiographic assessment of the spine in trauma. ${ }^{15}$ The committee divided patients into three groups: (1) awake, asymptomatic patients; (2) awake, symptomatic patients and (3) obtunded patients. There are different imaging and initial treatment algorithms for patients falling into each category, supported by class I evidence. Awake patients with no neurological symptoms or neck pain, in the absence of distracting injuries, who can perform a full neck range of motion without pain, do NOT require imaging or continued cervical spine immobilisation. This guideline was investigated by the National Emergency X-Radiography Utilization Study Group (NEXUS). Their trial prospectively studied a total of 34069 blunt trauma patients of whom 4309 were asymptomatic. ${ }^{16}$ All patients underwent standard three-view cervical spinal radiographs supplemented with CT as needed. Five criteria had to be met for the patient to be classified as having a low probability of injury: no midline cervical tenderness, no focal neurological deficit, normal alertness, no intoxication and no painful distracting injury. These criteria alone identified 810 of the 818 patients who had a cervical spinal injury, with a sensitivity of $99 \%$. Although not $100 \%$ sensitive, clinicians can easily apply the NEXUS criteria and these should serve as a guideline when deciding whether to request further cervical spine imaging for an awake and asymptomatic patient.

In awake but symptomatic patients, traditional three-view radiographs (anteroposterior, lateral and open-mouth odontoid view) should be obtained ONLY if it is not possible to obtain a high-quality CT scan. If CT imaging is readily available, as it is in nearly all trauma centres, then CT of the cervical spine should be the initial imaging study. If the CT 
scan is normal and the patient continues to have neck pain, then MR scanning is appropriate, specifically with short T1 inversion recovery (STIR) sequences. This STIR sequence is a fat-suppression method that better delineates soft-tissue injury. Damaged ligaments could signify a laxity in the joints and vertebrae, which could cause a subluxation and encroachment of the spinal canal. The clinician must remember that MRI evidence of ligamentous injury is best appreciated within $48 \mathrm{~h}$ of the injury and does not necessarily translate to true spinal instability, as it is a surrogate for pathological bony movement and may give false positives. ${ }^{17}$ True cervical spinal instability can be directly evaluated with cervical flexion-extension lateral radiographs. These films must be performed under controlled conditions to ensure that the patient does not move his/her neck past the point of worsening pain or symptoms, and the lateral views must include the C7-T1 disc space to ensure the entire cervical spine can be imaged.

There is still debate regarding the proper treatment if MRI and/or flexion-extension radiographs do not show pathology in a symptomatic patient. While it is probably safe to remove a cervical collar after negative imaging, we advocate continued use of the collar until the patient is asymptomatic. Many patients experience neck pain from muscle spasm or soft tissue trauma. This pain does not necessarily indicate an occult fracture or ligamentous injury and usually resolves over a few weeks. We re-evaluate this type of patient 2-4 weeks after the injury, and if the pain is resolved and if the patient has a stable and normal neurological examination, then we remove the collar without further imaging. If, however, pain persists past this point-less indicative of muscle strain-then we would consider repeating the dynamic X-rays.

Obtunded or comatose patients present a dilemma in determining the diagnosis and best further management. The useful NEXUS criteria cannot be applied to a patient for whom we cannot get reliable examination results, and therefore imaging evaluation becomes even more important in establishing a diagnosis. These patients should have a high-quality CT scan of the entire spinal axis as there is a risk of noncontiguous injury that would otherwise remain occult. If the CT scan is normal, MR imaging within $48 \mathrm{~h}$ may identify subtle signs of cervical spine injury. If the MR scan is normal or if the scan cannot be performed within $48 \mathrm{~h}$, the clinician must determine whether to continue cervical collar immobilisation on an individual patient basis.

\section{MEDICAL AND PHARMACOLOGICAL MANAGEMENT}

There is strong evidence from physiological animal studies to show that hypotension and hypoxaemia both contribute to secondary injury after an SCI. As occurs with head injury, the spinal cord loses the ability to autoregulate after injury, and vasoreactivity may contribute to local hypoperfusion. This can be significantly exacerbated by spinal shock, which leads to the loss of peripheral vascular tone (among other effects) and further hypotension and hypoperfusion. This may lead to increased secondary damage to the spinal cord around the site of injury in the hours and days after the trauma. Therefore, clinicians must focus on avoiding hypoxia and hypotension in the acute postinjury period: this can be best achieved in an intensive care unit. ${ }^{18}$ Patients with high-cervical SCI require careful airway management with careful and expedient intubation. Avoiding catastrophic airway loss is critical in the acute postinjury period. ${ }^{19}$ Careful ventilator and airway management should reduce the chance of pneumonia in this precarious patient population.

There is still controversy regarding blood pressure management in acute cervical SCI. The abundant class III evidence suggests that an elevated mean arterial pressure over 85 or $90 \mathrm{~mm} \mathrm{Hg}$ results in independently improved outcomes. ${ }^{20-22}$ The retrospective nature of the data in the literature regarding optimal duration of treatment and the target mean arterial pressure makes elevation of this pressure in patients with SCI purely a recommendation rather than a steadfast guideline. We believe that future studies will lend more concrete credence to this approach. ${ }^{23}$ We generally treat patients with cervical SCI with norepinephrine for 7 days after the injury to maintain a mean arterial pressure between 85 and $90 \mathrm{~mm} \mathrm{Hg}$. The aggressiveness of this treatment is anecdotally titrated based on the severity of the patient's injury and any associated or pre-existing cardiopulmonary comorbidities.

The use of corticosteroids in acute SCI has been even more controversial in recent decades than optimal blood pressure management. Corticosteroids, specifically methylprednisolone, received an enormous amount of attention in the 1990 s as a powerful neuroprotective agent. National Acute Spinal Cord Injury Study (NASCIS) II and III were pivotal studies in guiding current treatment. ${ }^{24}{ }^{25}$ Both NASCIS II and II were designed as class I prospective, randomised trials; but their conclusions on the benefit of corticosteroid treatment did not bear out through post hoc analysis, thereby downgrading the level of evidence. Furthermore, these studies showed a trend towards significantly increased morbidity and mortality rates in patients treated with corticosteroids, specifically for pneumonia, sepsis, acute respiratory distress syndrome, gastrointestinal haemorrhage and death. ${ }^{26}$ Given the entirety of the current literature on corticosteroid treatment for acute SCI, we do not advocate its use given the significant increase in complications and lack of clearly defined benefits. 


\section{CLASSIFICATION OF CERVICAL SPINE INJURIES}

As previously described, cervical SCI may be complete or incomplete, with some preserved neurological function across the site of injury. Incomplete SCIs often fall into described patterns, such as BrownSéquard syndrome, central cord syndrome or anterior or posterior cord syndrome, based on the mechanism and location of injury within the cord. The symptoms and findings associated with each of these syndromes exist on a spectrum. ${ }^{27}$

Osseous injury often accompanies cervical SCI. The following paragraphs briefly review common osseous injuries and their classifications.

Trauma at the skull base can fracture the occipital condyle or lead to occipitocervical dislocation. Occipital condyle fractures are typically classified into one of three categories. Class I is a comminuted fracture of the condyle caused by impaction from the lateral mass of C1. Class II involves a related basilar skull fracture. Class III is an avulsion fracture at the site of the attachment of the alar ligament. ${ }^{28}$

Occipitocervical dislocation usually results from a high-energy trauma and is often fatal. These injuries are also typically categorised into one of three categories. Type I involves a ventral subluxation of the condyles relative to the $\mathrm{C} 1$ lateral masses. Type II involves a vertical dislocation of the occipital condyles (figure 1). Type III injuries are rare and involve dorsal dislocations of the condyles. ${ }^{29}$

Fractures of C1 usually fall into one of three categories. Type I fractures are limited to the dorsal arch of C1. Type II injuries involve a unilateral lateral mass injury. Type III injuries, classically called 'Jefferson fractures', are burst-type fractures with three or more fracture sites through the ventral and dorsal aspects of

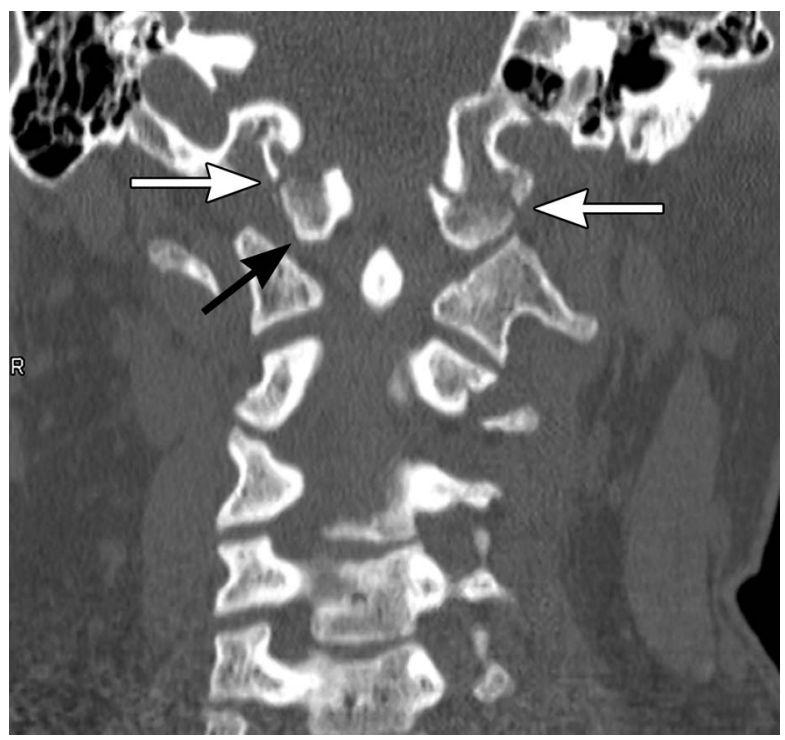

Figure 1 Coronal CT scan shows a type II occipitocervical dislocation (black arrow) with associated bilateral fractures of the occipital condyles (white arrows). Copyright: Barrow Neurological Institute.

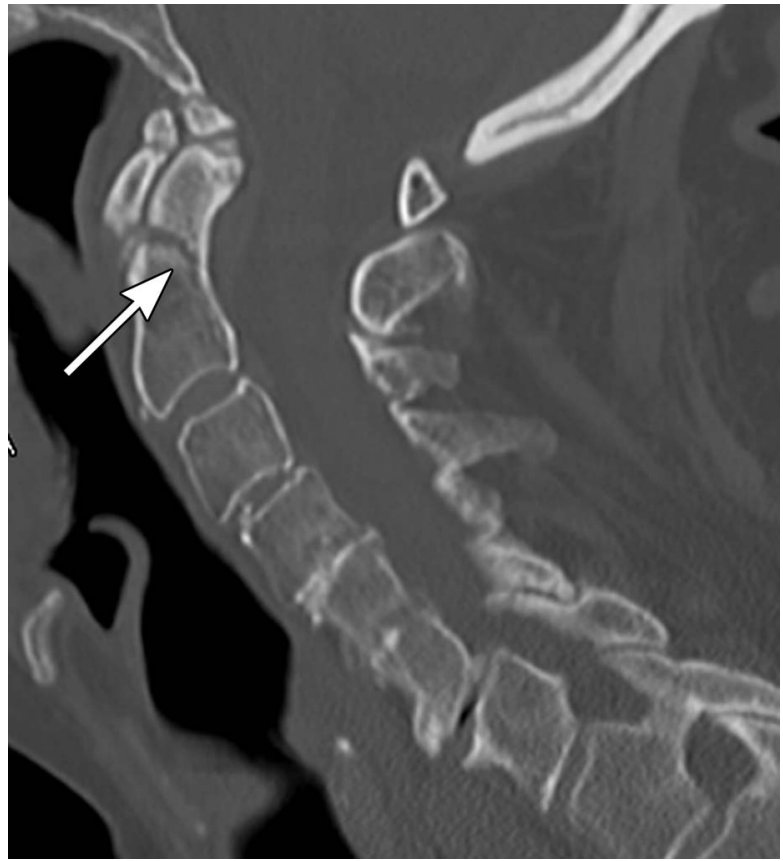

Figure 2 Sagittal CT scan shows a type II odontoid (C2) fracture with mild angulation of the fracture (arrow). Copyright: Barrow Neurological Institute.

the $\mathrm{C} 1$ ring. $\mathrm{C} 1$ fractures may be associated with disruption of the transverse atlantal ligament; this ligament's incompetence results in an unstable C1-C2 articulation. $^{30}$

C2 fractures are of two common types, including fractures of the odontoid process or pars interarticularis. Odontoid fractures are most commonly classified by the anatomical location of the fracture. Type I fractures occur at the superior tip of the dens. Type II fractures occur at the junction of the base of the dens and the body of the axis (figure 2). Type III fractures extend into the body of the axis. ${ }^{31}$ Fractures of the pars interarticularis, also called 'hangman's fractures', also are commonly organised into three categories. Type I fractures have $<3 \mathrm{~mm}$ of translation of $\mathrm{C} 2$ on $\mathrm{C} 3$ and lack significant angulation at the fracture site. Type II fractures have $>3 \mathrm{~mm}$ of translation and significant angulation (figure 3). Type III fractures involve pars fractures plus bilateral $\mathrm{C} 2 / 3$ facet dislocations. $^{32}$

Subaxial cervical spine injuries are typically classified by mechanism. The C3-C7 vertebrae are similar in anatomy and biomechanics. These vertebrae sustain similar fracture patterns. Six common fracture patterns include compressive flexion (figure 4), compressive extension, distractive flexion, vertical compression, distractive extension and lateral flexion. Treatment for these injuries is tailored to the severity of the osseous and ligamentous injury.

\section{SURGICAL PRINCIPLES}

Many factors influence the need for surgery in acute traumatic cervical spine injury. The massive advances 


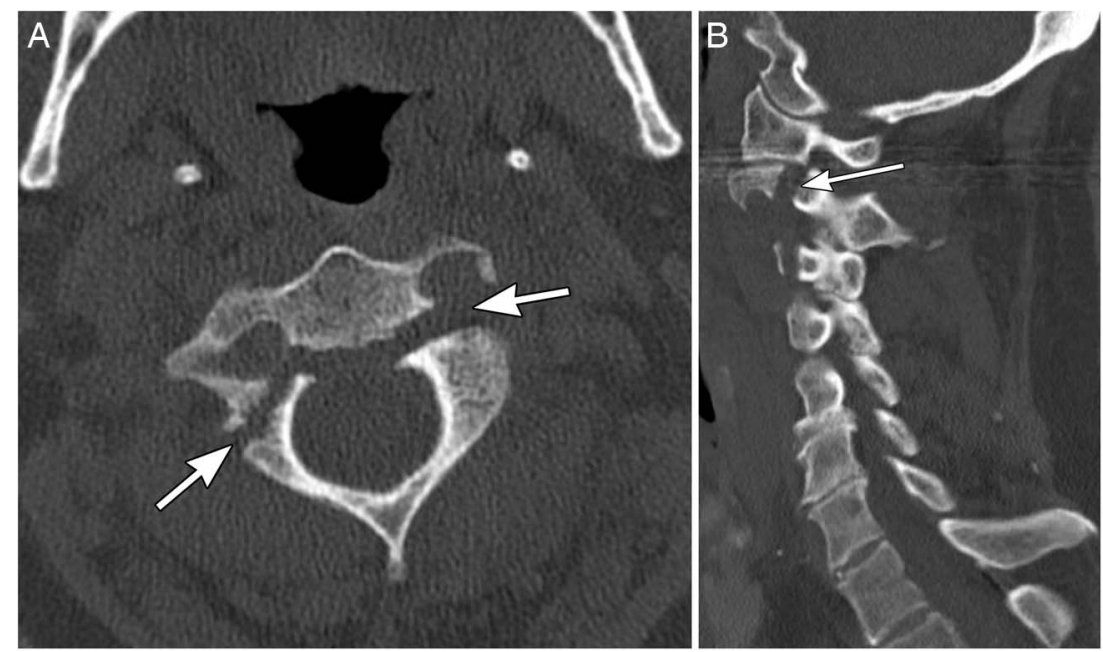

Figure 3 A type Il hangman's fracture of the pars (arrows) on (A) axial and (B) sagittal CT scans (arrows). Copyright: Barrow Neurological Institute.

in instrument technology have made possible effective fixation and fusion of nearly all traumatic spinal injuries. The combination of decompression of injured neural elements with correction of deformity,

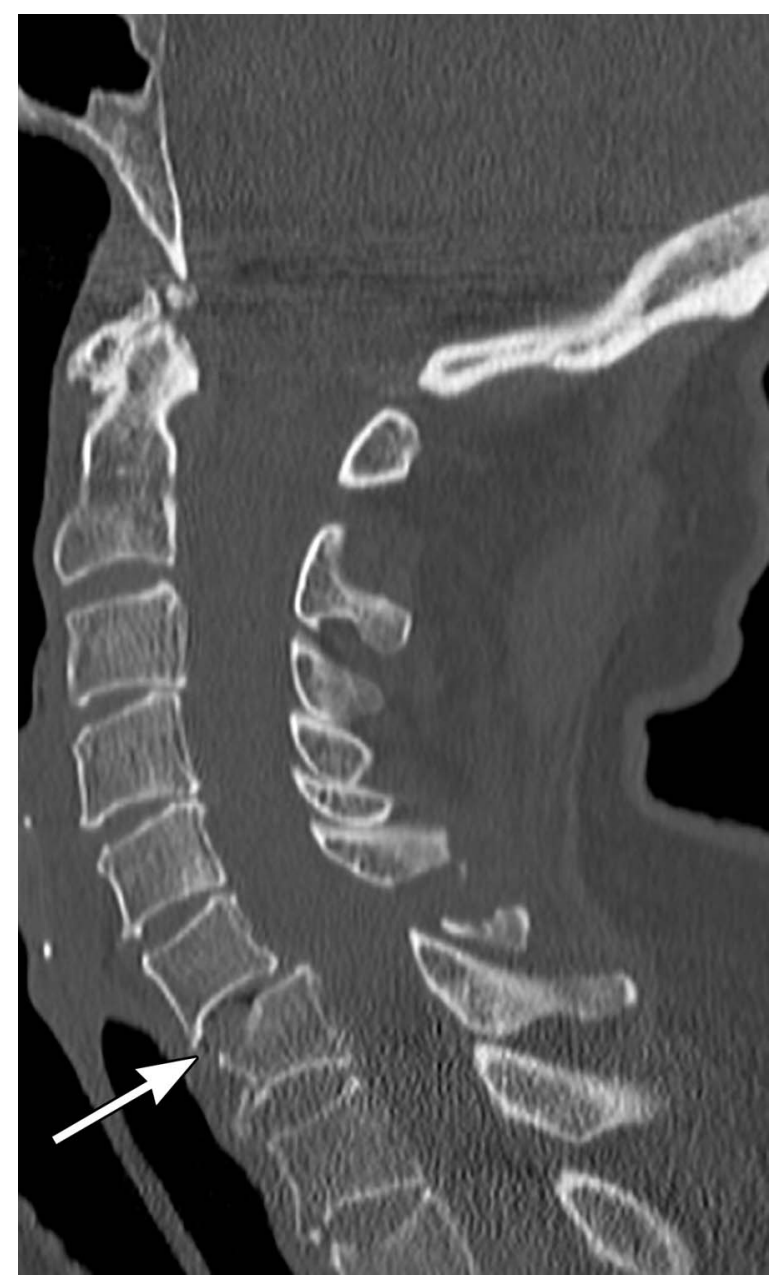

Figure 4 Sagittal CT scan shows a compressive flexion fracture of the C7 vertebral body (arrow) with associated posterior element fractures. Copyright: Barrow Neurological Institute. reduction of fractures and fusion for long-term spinal stability are all elements of a successful spinal trauma surgery. Surgeons must ensure adequate removal of bone and ligament to relieve any compression on the neural elements. One aspect of cervical spine instrumentation to emphasise is the importance of both fixation and fusion. These terms should not be used interchangeably. Fixation refers to the instrumentation, such as lateral mass screws that are linked with top-loading rods. This titanium hardware serves as 'rebar' or a scaffold to fixate unstable segments. This hardware provides short-term stability and prevents motion across the involved segments. Fusion refers to the bony mass that will ultimately provide long-term stability across segments, much like concrete used with rebar. Fusion is created by decorticating the native bone-especially in the facets or disc spaces (for anterior approaches) that are the natural joints of the cervical spine. These spaces are then filled with either the patient's own autograft or allograft bone (or both). The goal is to create a bony fusion across motion segments, creating one large functional bone where there were once multiple bones. The fusion process may take up to 1 year to complete and is aided by direct loading (a tenet known as Wolff's law) and by immobilisation. The specific procedures used for cervical fixation and fusion are beyond the scope of this review. However, stabilising procedures may be performed through either an anterior or a posterior approach. The choice of procedure that might best benefit a specific patient is determined by the injury pattern, comorbidities, area of most significant compression, type of deformity and surgeon preference.

There is also controversy with regard to the timing of surgery. While some surgeons advocate early decompression to minimise the time of spinal cord compression, the optimal timing for decompression has yet to be established in a randomised, prospective fashion. The recent Surgical Timing in Acute Spinal 
Cord Injury Study showed improved outcomes at 6 months with early ( $<24$ h from injury) when compared with late intervention $(\geq 24 \mathrm{~h}) .{ }^{33}$ While early spinal decompression may provide neurological benefit, spinal stabilisation generally allows the patient to mobilise earlier. Many surgeons feel that this mobilisation decreases early complications. While there is no class I evidence for early decompression, we believe that this is a priority in SCI to maximise the chances and degree of recovery.

\section{CONCLUSIONS}

Spinal cord injuries remain a clinical challenge. The evaluation, classification and initial medical management of patients with these injuries have become increasingly standardised. Considerable resources are being committed to improving both medical and surgical treatment options. Despite the progress that has been made in the acute management of patients with SCI, neurological outcomes have not improved significantly in recent decades. Long-term management strategies, including rehabilitation, will remain critical in optimising outcomes. Better understanding of SCI pathophysiology will ultimately provide the basis for novel treatment strategies with the eventual goal of improving neurological function to the preinjury baseline.

\section{Key messages}

- Trauma patients suspected of having spine injuries should be properly immobilised before transport to the hospital.

- Clinicians should familiarise themselves with the ASIA and SCIM grading scales for spinal cord injuries, as they offer a reproducible way to communicate and track the neurological function of these patients.

- In awake and asymptomatic trauma patients, cervical spine imaging is not needed if patients meet the NEXUS criteria for low risk of injury.

- Patients with a spinal cord injury should receive permissive hypertension (mean arterial pressure $>85$ $\mathrm{mmHg}$ ) for 7 days after the injury.

- Although the role of corticosteroids in the acute management of traumatic spinal cord injury is still controversial, we do not advocate their use.

Acknowledgements We thank the Barrow Neurological Institute Neuroscience Publications office for assistance with document and figure preparation.

Contributors AER and NT conceived the idea of the study and were responsible for the design of the study. AER and MTN were responsible for undertaking for the data analysis and produced the tables and graphs. AER, MTN and NT provided input into the data analysis. The initial draft of the manuscript was prepared by AER and MTN and then circulated repeatedly among all authors for critical revision. All authors were responsible for the acquisition of the data and all contributed to the interpretation of the results.
Competing interests None declared.

Provenance and peer review Commissioned; externally peer reviewed. This paper was reviewed by Martin Wilby, Liverpool, UK and George Malcolm, Bristol, UK.

\section{REFERENCES}

1 Snyder EY, Teng YD. Stem cells and spinal cord repair. N Engl J Med 2012;366:1940-2.

2 [No authors listed]. Spinal cord injury facts and figures at a glance. J Spinal Cord Med 2014;37:479-80.

3 Devivo MJ. Epidemiology of traumatic spinal cord injury: trends and future implications. Spinal Cord 2012;50: 365-72.

4 Facts and figures. Secondary facts and figures. http://www. spinal-research.org/research-matters/spinal-cord-injury/ facts-and-figures

5 Theodore N, Hadley MN, Aarabi B, et al. Prehospital cervical spinal immobilization after trauma. Neurosurgery 2013;72 (Suppl 2):22-34.

6 Del Rossi G, Heffernan TP, Horodyski M, et al. The effectiveness of extrication collars tested during the execution of spine-board transfer techniques. Spine J 2004;4:619-23.

7 Burton JH, Dunn MG, Harmon NR, et al. A statewide, prehospital emergency medical service selective patient spine immobilization protocol. J Trauma 2006;61:161-7.

8 Haut ER, Kalish BT, Efron DT, et al. Spine immobilization in penetrating trauma: more harm than good? J Trauma 2010;68:115-20; discussion 20-1.

9 Bauer D, Kowalski R. Effect of spinal immobilization devices on pulmonary function in the healthy, nonsmoking man. Ann Emerg Med 1988;17:915-18.

10 Liew SC, Hill DA. Complication of hard cervical collars in multi-trauma patients. Aust N Z J Surg 1994;64:139-40.

11 Davies G, Deakin C, Wilson A. The effect of a rigid collar on intracranial pressure. Injury 1996;27:647-9.

12 Maynard FM Jr, Bracken MB, Creasey G, et al. International Standards for Neurological and Functional Classification of Spinal Cord Injury. American Spinal Injury Association. Spinal Cord 1997;35:266-74.

13 Catz A, Itzkovich M, Agranov E, et al. SCIM-spinal cord independence measure: a new disability scale for patients with spinal cord lesions. Spinal Cord 1997;35:850-6.

14 Catz A, Itzkovich M, Tesio L, et al. A multicenter international study on the Spinal Cord Independence Measure, version III: Rasch psychometric validation. Spinal Cord 2007;45:275-91.

15 Ryken TC, Hadley MN, Walters BC, et al. Radiographic assessment. Neurosurgery 2013;72(Suppl 2):54-72.

16 Hoffman JR, Mower WR, Wolfson AB, et al. Validity of a set of clinical criteria to rule out injury to the cervical spine in patients with blunt trauma. National Emergency X-Radiography Utilization Study Group. N Engl J Med 2000;343:94-9.

17 Horn EM, Lekovic GP, Feiz-Erfan I, et al. Cervical magnetic resonance imaging abnormalities not predictive of cervical spine instability in traumatically injured patients. Invited submission from the Joint Section Meeting on Disorders of the Spine and Peripheral Nerves, March 2004. J Neurosurg Spine 2004;1:39-42.

18 Casha S, Christie S. A systematic review of intensive cardiopulmonary management after spinal cord injury. J Neurotrauma 2011;28:1479-95.

19 Hassid VJ, Schinco MA, Tepas JJ, et al. Definitive establishment of airway control is critical for optimal 
outcome in lower cervical spinal cord injury. J Trauma 2008;65:1328-32.

20 Vale FL, Burns J, Jackson AB, et al. Combined medical and surgical treatment after acute spinal cord injury: results of a prospective pilot study to assess the merits of aggressive medical resuscitation and blood pressure management. J Neurosurg 1997;87:239-46.

21 Levi AD, Tator CH, Bunge RP. Clinical syndromes associated with disproportionate weakness of the upper versus the lower extremities after cervical spinal cord injury. Neurosurgery 1996;38:179-83; discussion 83-5.

22 Dolan EJ, Tator $\mathrm{CH}$. The effect of blood transfusion, dopamine, and gamma hydroxybutyrate on posttraumatic ischemia of the spinal cord. J Neurosurg 1982;56:350-8.

23 Ryken TC, Hurlbert RJ, Hadley MN, et al. The acute cardiopulmonary management of patients with cervical spinal cord injuries. Neurosurgery 2013;72(Suppl 2):84-92.

24 Bracken MB, Shepard MJ, Collins WF, et al. A randomized, controlled trial of methylprednisolone or naloxone in the treatment of acute spinal-cord injury. Results of the Second National Acute Spinal Cord Injury Study. N Engl J Med 1990;322:1405-11.

25 Bracken MB, Shepard MJ, Holford TR, et al. Administration of methylprednisolone for 24 or 48 hours or tirilazad mesylate for 48 hours in the treatment of acute spinal cord injury. Results of the Third National Acute Spinal Cord Injury Randomized Controlled Trial. National Acute Spinal Cord Injury Study. JAMA 1997;277:1597-604.

26 Hurlbert RJ, Hadley MN, Walters BC, et al. Pharmacological therapy for acute spinal cord injury. Neurosurgery 2013;72 (Suppl 2):93-105.

27 Nowak DD, Lee JK, Gelb DE, et al. Central cord syndrome. J Am Acad Orthop Surg 2009;17:756-65.

28 Karam YR, Traynelis VC. Occipital condyle fractures. Neurosurgery 2010;66(3 Suppl):56-9.

29 Montane I, Eismont FJ, Green BA. Traumatic occipitoatlantal dislocation. Spine (Phila Pa 1976) 1991;16:112-16.

30 Kesterson L, Benzel E, Orrison W, et al. Evaluation and treatment of atlas burst fractures (Jefferson fractures). J Neurosurg 1991;75:213-20.

31 Anderson LD, D'Alonzo RT. Fractures of the odontoid process of the axis. J Bone Joint Surg Am 1974;56:1663-74.

32 Rayes M, Mittal M, Rengachary SS, et al. Hangman's fracture: a historical and biomechanical perspective. J Neurosurg Spine 2011;14:198-208.

33 Fehlings MG, Vaccaro A, Wilson JR, et al. Early versus delayed decompression for traumatic cervical spinal cord injury: results of the Surgical Timing in Acute Spinal Cord Injury Study (STASCIS). PLoS ONE 2012;7:e32037. 